\title{
The Use of Gyroscope Feedback in the Control of the Walking Gaits for a Small Humanoid Robot
}

\author{
Jacky Baltes, Sara McGrath, and John Anderson \\ Department of Computer Science, \\ University of Manitoba, \\ Winnipeg, Canada \\ jacky@cs.umanitoba.ca \\ http: //www.cs.umanitoba.ca/ jacky
}

\begin{abstract}
This paper describes methods used in stabilizing the walking gait of TAO-PIE-PIE, a small humanoid robot given rate feedback from two RC gyroscopes. TAO-PIE-PIE is a fully autonomous small humanoid robot $(30 \mathrm{~cm}$ tall). Although TAO-PIE-PIE uses a minimal set of actuators and sensors, it has proven itself in international competitions, winning honors at the RoboCup and FIRA HuroSot competitions in 2002 and 2003. The feedback control law is based solely on the rate information from two RC gyroscopes. This alleviates drift problems introduced by integrating the $\mathrm{RC}$ gyroscope feedback in the more common position control approaches.
\end{abstract}

\section{Introduction}

Recent years have seen increased interest in humanoid robots, with many small humanoid robots emerging from research labs, hobbyists, and universities mainly in Japan, but also other countries ([4], [5], [3]).

This paper describes our first attempts at using feedback control to balance the walking gait of TAO-PIE-PIE. TAO-PIE-PIE was intended as a research vehicle to investigate methods for deriving control methods for stable walking patterns for humanoid robots. Stable walking, especially over uneven terrain, is a difficult problem. One problem is that current actuator technology (RC Servos, DC motors) generate less torque in comparison to their weight than human muscle. Another problem is that feedback from gyroscopes and actuators is very noisy. The necessary smoothing of the input signals makes it hard to use them in actively controlling the walking motion.

Cost was an important design criteria in TAO-PIE-PIE's development. Previous experience has shown us that the use of commonly available cheap components not only helps to keep the cost of a project down, but it also has led to the development of novel, versatile, and robust approaches to problems in robotics.

Another design goal was to reduce the number of degrees of freedom (DOF) of the robot. This reduces the cost of the humanoid robot as well as increases its robustness. Each DOF adds extra complexity in the mechanical design and the design of the control electronics. Furthermore, reducing the number of DOFs allows us to exploit the dimensions of the humanoid walking problem. The minimum set of DOFs that allow a humanoid robot to walk is also of interest, since it leads to energy efficient designs. 

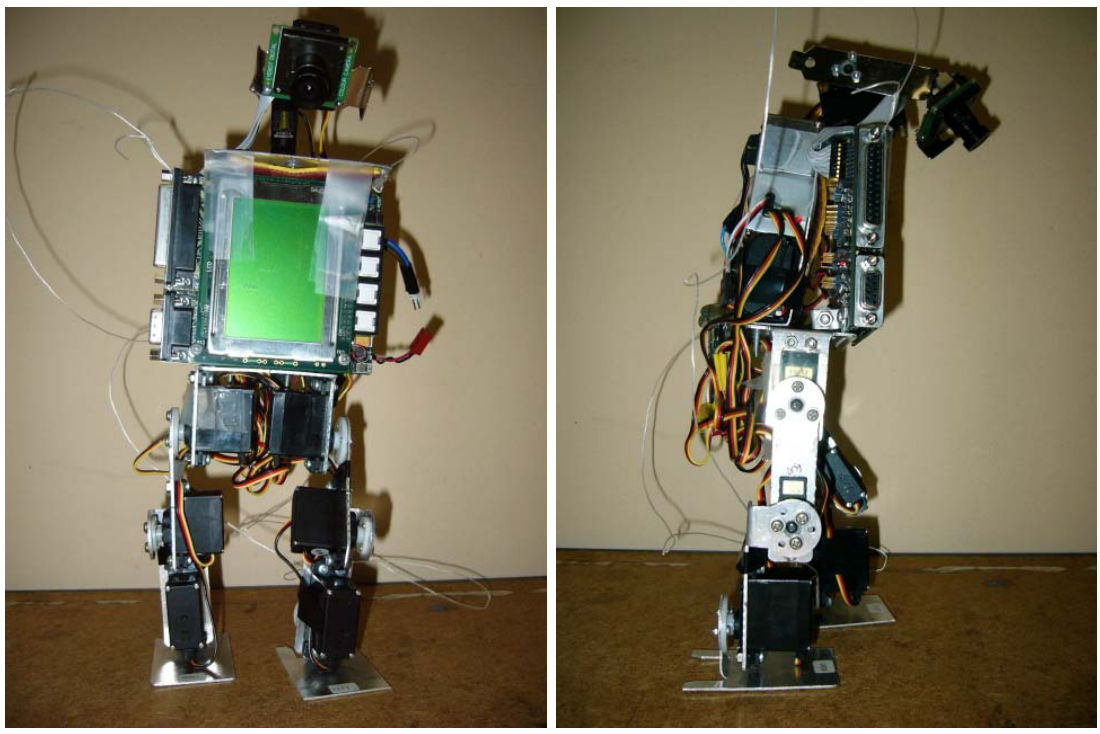

Fig. 1. Front and side view of TAO-PIE-PIE

TAO-PIE-PIE is the third generation of humanoid robots developed in our lab. Figure 1 shows the mechanical construction of TAO-PIE-PIE.

The actuators and sensors consist of widely available RC servos and RC gyroscopes for remote controlled cars and helicopters.

The Eyebot controller ([2]) was chosen as embedded processor, since it is relatively inexpensive, yet powerful enough to provide vision information. A small CMOS camera provides visual feedback for the robot.

The mechanical design was done in conjunction with Nadir Ould Kheddal's robotics group at Temasek Politechnic, Singapore. TAO-PIE-PIE is constructed out of $0.5 \mathrm{~mm}$ aluminum, with RC servos used as structural components in the design.

Furthermore, TAO-PIE-PIE is intended to compete at international humanoid robotic competitions such as RoboCup and FIRA HuroSot ([1]. Among other things, this means that TAO-PIE-PIE must be able to actively balance, walk, run an obstacle course, dance, and kick a ball.

The remainder of this paper is structured as follows. The methodology used to develop and details of the implementation of the walking gaits are given in section 2, while section 3 presents an evaluation of this approach.

\section{The Walking Gait}

One of the fundamental problems in humanoid robots is the development of stable walking patterns. A walking pattern is dynamically stable if the center of pressure (COP) is within the supporting area. A statically stable walking pattern also has the center of mass (COM) within the supporting area. 


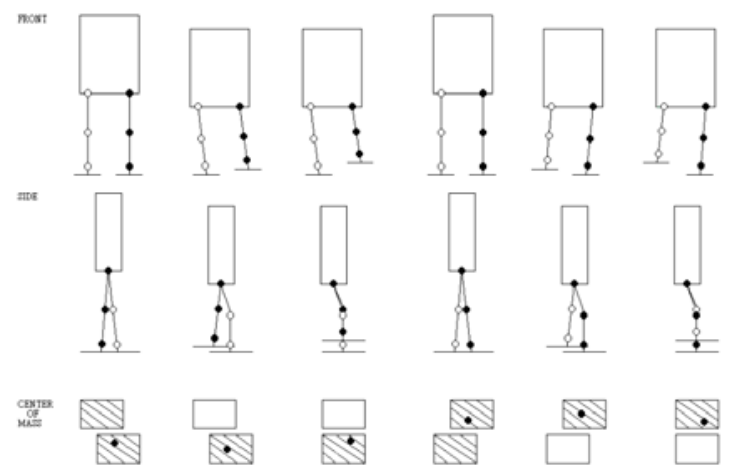

Fig. 2. Walking Pattern of TAO-PIE-PIE

We employ a divide and conquer approach and partition the statically stable walking gaitinto six phases: three for the right leg and three for the left. The phases were selected in such a way that the robot is statically stable at the end of eachphase.

The six phases of the walking pattern for a straight walk is shown in Figure 2, The bottom row of images in Figure 2 shows the approximate position of the COM in each phase. We describe these phases moving from left to right in the figure.

TAO-PIE-PIE starts in phase 1 — "Two Leg Stand" — where the right leg is in front and the left leg is behind. Both legs are on the ground and the COM is between the two legs.

From phase 1, TAO-PIE-PIE moves to phase 2 - "One Leg Stand" - In this phase, the ankle servo generates a torque which moves the COM to the inside edge of the right leg. This also results in the back (left) leg to lift off the ground.

During the transition from phase 2 to phase 3 - "Ready for Landing" — is in static balance. TAO-PIE-PIE moves the free left leg forward and positions it so that it is ready for landing. The COM moves to the front of the supporting leg. This stabilizes the transition to phase 4.

During the transition from phase 3 to phase 4 - "Two Leg Stand Inverse" — the robot is in dynamic balance. The supporting leg extends its knee joint to shift the COM over the front edge of the supporting leg. The ankle servo of the supporting leg generates a torque to move the COM over the right side. The left leg will touch the ground in front of the right leg.

Phases 5 and 6 are the mirror images of phases 2 and 3 respectively. After phase 6 , the motion continues with a transition to phase 1 .

\subsection{Sensor Feedback}

The only feedback about the motion of TAO-PIE-PIE is provided by two gyroscopes that provide information about the angular velocity in the lateral plane and saggital plane respectively.

The raw sensor data of the gyroscopes is very noisy. We therefore compute a running average over five samples to smooth out the noise. Figure 3 shows the gyroscope readings for the lateral and saggital plane over approximately twenty steps. 

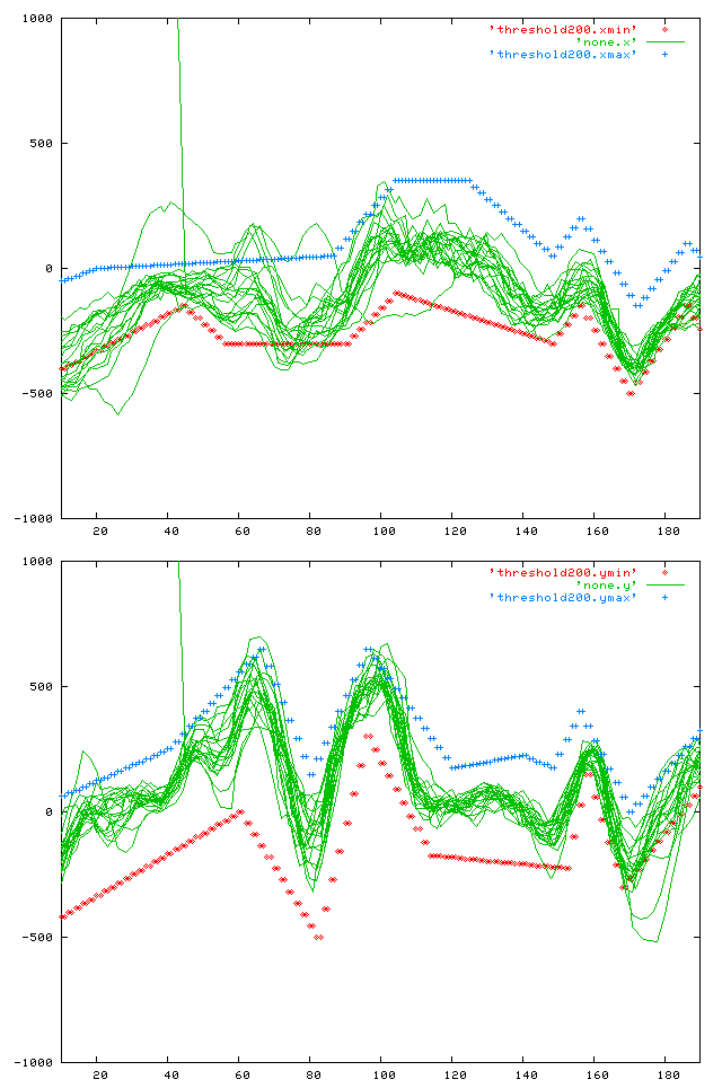

Fig. 3. Gyroscope Readings in the lateral and saggital plane over 10 Steps. Linear Approximation of the Safe Zone

Since TAO-PIE-PIE did not fall over during this extended walking trial, these gyroscope readings were used to determine a "safe zone" for the velocity feedback of the gyroscopes.

We then created a linear approximation of the "safe zone envelope" and generated minimum and maximum thresholds for the gyroscope readings. The approximation is shown using red and blue lines in Fig. 3 .

\subsection{Sensor Feedback in Detecting a Fall}

Initially, we ran a series of experiments to verify the accuracy of the approximated "safe zone" by making TAO-PIE-PIE beep whenever the measured angular velocity was above or below the threshold in the saggital and lateral plane respectively. The goal was to show that TAO-PIE-PIE would beep just before falling over. These experiments proved very successful. TAO-PIE-PIE detected a fall with $95 \%$ accuracy with few $(<5 \%)$ false positives. 


\subsection{Motion Compensation}

After verifying that the gyroscope data can be used to predict a fall for TAO-PIE-PIE, the next step was to develop a method for modifying the motion parameters to avoid a fall. There are three inputs to the motion compensation algorithm:

1. Saggital plane gyroscope reading;

2. Lateral plane gyroscope reading; and

3. The current phase of the walk.

Initially, the most common cause for TAO-PIE-PIE falling over was a fall to the right in phase 2 (see Fig. 2) or to the left in phase 5. This is due to the fact that because of the limited number of DOFs, TAO-PIE-PIE uses the ankle servo to move the COM over the right or left foot. Since the torso of TAO-PIE-PIE is fixed, TAO-PIE-PIE is precariously balanced at this point and the robot sometimes moves to far, resulting in a fall to the right or left respectively.

The first motion compensation algorithm is active when the lateral plane gyroscope reading is larger/smaller than the maximum/minimum velocity threshold in phase $2 / 5$ respectively. In this case, the robot tends to fall towards the right/left.

There are two ways in which the rotational velocity in the saggital plane can be controlled:

1. The set point for the right or left ankle servo can be changed to induce a torque in the opposite direction to the fall;

2. The robot can extend the knee and hip joint, resulting in a slowed down rotation. This effect is similar to the effect of slowing down the rotation of a chair while seated in it by extending one's arms.

We focus on modifying the angular velocity through the first method, since during a straight walk, the left-right velocity is mainly generated through the ankle servos. The second method is disadvantageous in that it also modifies the forward-backward balance of the robot. The set points for the servos are based on linear interpolations between a set of control points.

If the angular velocity is too large, then the motion compensator modifies the set point of the servo by moving it $10 \%$ closer to the start point of the pattern. Similarly, if the angular velocity is not large enough, then the set point is slightly extended.

The same approach is used when controlling falls in the saggital plane. In this case, however, there is no single servo that is responsible for the angular velocity. Instead, both set points for the knee and hip joint are modified by $90 \%$ to prevent a fall.

The feedback from the gyroscopes is also used to detect abnormal behavior. For example, if the robot's foot is caught on the carpet, instead of moving the leg forward, the robot will fall onto the leg too early. If this abnormal feedback is detected the robot attempts to stabilize itself by constraining all movement within the phases, in essence putting both feet on the ground as quickly as possible and straightening up its upper body. The constrainment will continue until both gyroscopes show appropriate angular velocities. 


\section{Evaluation}

We evaluated the motion compensation algorithm by subjectively looking at the static walking pattern. The standard walking pattern of TAO-PIE-PIE is quite stable even without motion compensation. The robot did not fall during any of these experiments. However, the walking gait with motion compensation was more balanced resulting in a straight line walk. Without motion compensation, TAO-PIE-PIE would veer to the right significantly. The walking speed of the robot remains unchanged.

We also evaluated the motion compensation by subjectively by comparing the gyroscope feedback with and without motion compensation. The results of this comparison are shown in Fig. 4
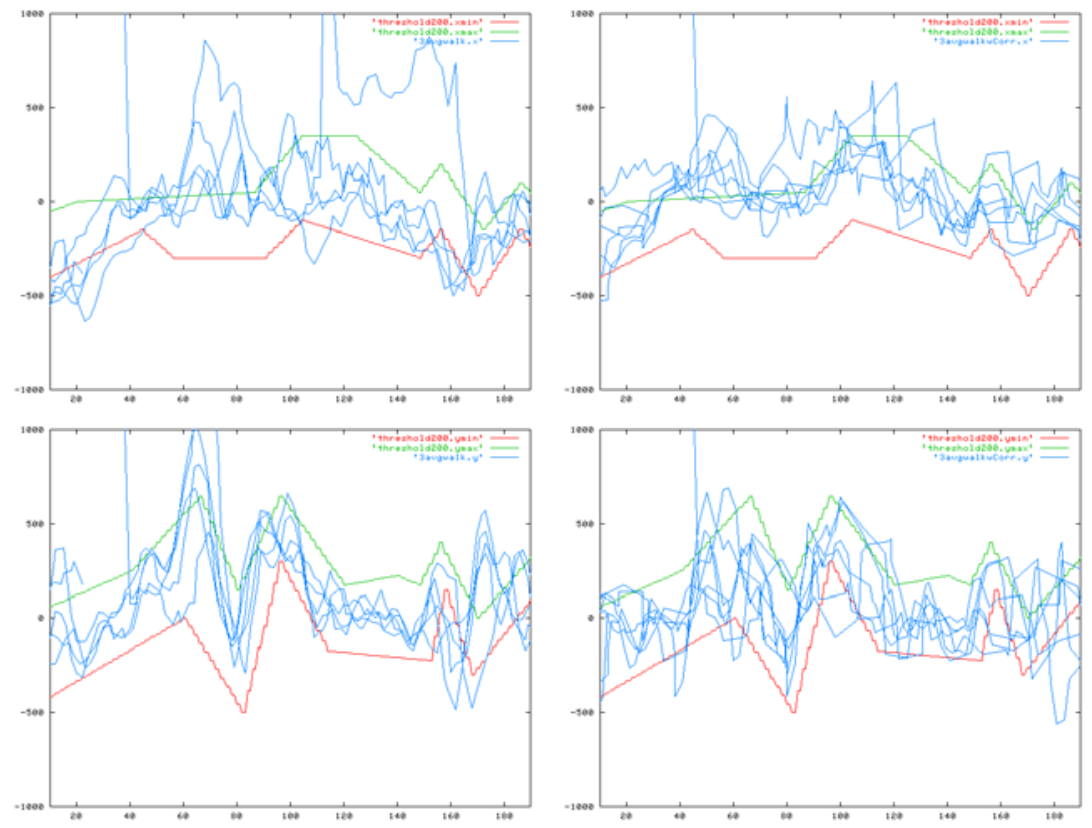

Fig. 4. Comparison of original walking gait (left column) and walking gait with motion compensation (right column) in the saggital (top row) and lateral (bottom row) plane

As can be seen from the plots, the motion compensation does constrain the walking gait so that the gyroscope feedback is more in the desired envelope. Most of the time, the walking gait remains in the desired velocity envelope.

Work is currently underway on developing a dynamic (shuffle-like) walk with correspondingly dynamic turns. When feedback correction was applied to walk, the walk covered more distance than without. Subjectively as well, the gyroscope feedback was not only better maintained within the desired envelope, but also formed a much more regular path, as shown in Fig. 5 . 

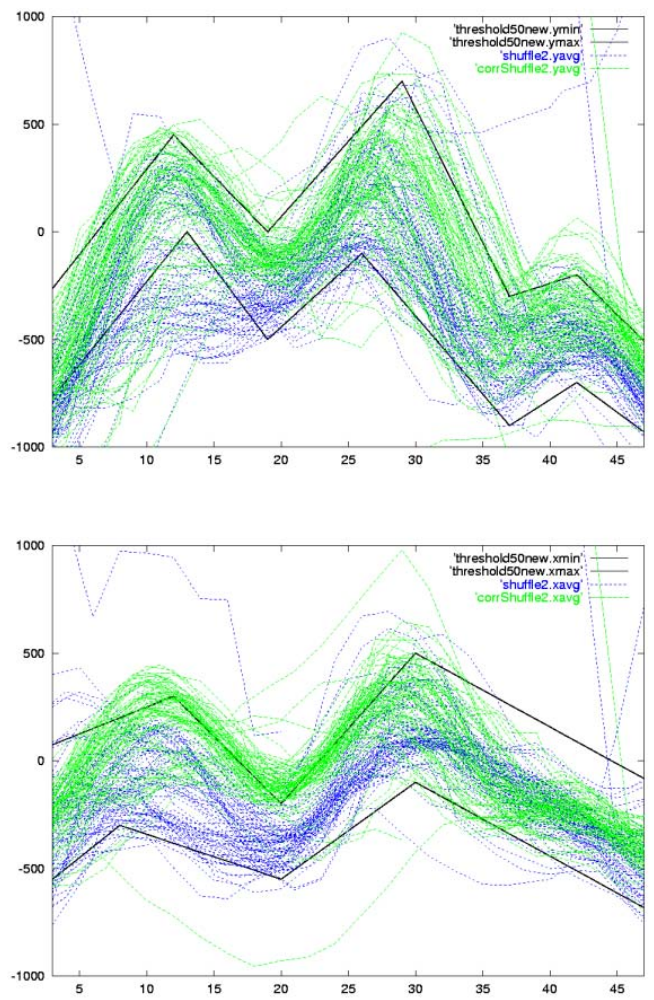

Fig. 5. Corrected and uncorrected dynamic walking gait in the saggital (top) and lateral (bottom) plane.

\section{Conclusion}

This paper describes our first experiments into the design of robust feedback control for walking of small humanoid robots. There is much work left to be done.

The current motion compensation algorithm is simple, but works surprisingly well in practice. We plan on investigating more complex methods for motion compensation and balancing in the future. For example, the motion compensation should not be a constant factor, but should be proportional to the current velocity.

We intend to extend this evaluation into more uneven terrains. The hope is that by using feedback, TAO-PIE-PIE is able to compensate for uneven terrain and adapt its walking gait. As well, these corrections could be applied to kicking and turning movements in order to correct for all movements the robot makes.

As noted in section 2, safe zones need to be developed from a previously stable walk. Thus speeding up a walk requires developing a stable walk in order to re-calibrate the safe envelopes. Investigation should proceed into the safe envelopes and their correlation to a change in speed or the desired walk pattern (fast, slow, left turn, right turn, etc). This 
could lead to implementing a gait or gait corrections without previously developing the stable walk.

TAO-PIE-PIE has shown itself to be a powerful and flexible platform for research into humanoid robotics. It has proven itself during international competitions winning a second place in theRoboCup anda technical merit award in the FIRA 2002 competitions. We have learned important lessons in the design of humanoid robots from TAO-PIE-PIE, which we will use in the design of the next generation humanoid robot Hiro. Hiro will use four additional DOFs (two in thehip and one for each leg). HIRo will also have more sensors,especially a set of force sensors in the feet. It also features a faster embedded processor (Intel Stayton), which allows us toimplementbetter on-board computer vision algorithms. One of the maingoals of the Hiro platform will be to investigate methods for augmenting the balancing of the robotusing visual feedback.

\section{References}

1. Jacky Baltes and Thomas Bräunl. HuroSot Laws of the Game. University of Manitoba, Winnipeg, Canada, May 2004. http://www.fira.net/hurosot.

2. Thomas Bräunl. Thomas bräunl's homepage. WWW, November 2002. http://robotics.ele.uwa.edu.

3. Jung-Hoon Kim, Ill-Woo Park, and Jun-Ho Oh. Design of a humanoid biped robot lower body. In Proceedings of the 3rd International Workshop on Human-friendly Welfare Robotic Systems. KAIST, January 20 - 222002.

4. Fuminori Yamasaki, Tatsuya Matsui, Takahiro Miyashita, , and Hiroaki Kitano. Pino the humanoid: A basic architecture. In Peter Stone, Tucker Balch, and Gerhard Kraetszchmar, editors, RoboCup-2000: Robot Soccer World Cup IV, pages 269-278. Springer Verlag, Berlin, 2001.

5. Changjiu Zhou. Linguistic and numeral heterogenous data integration with reinforcement learning for humanoid robots. In Proceedings of the 1st IEEE Conference on Humanoid Robots, 2000. 